\title{
ESTUDIO PALINOLÓGICO DEL YACIMIENTO ROMANO DE LA VEREDA (BURGUILLOS, SEVILLA): DE LA RECONSTRUCCIÓN PALEOAMBIENTAL A LA FUNCIONALIDAD DE LAS ESTRUCTURAS
}

\author{
PALYNOLOGICAL STUDY OF THE ROMAN SITE OF LA VEREDA (BURGUILLOS, SEVILLA): \\ FROM PALAEOENVIRONMENTAL RECONSTRUCTION TO FUNCTIONALITY OF STRUCTURES
}

\author{
SEBASTIÁN PÉREZ DÍAZ / DARÍO BERNAL CASASOLA² / JOSÉ ANTONIO LÓPEZ SÁEZ / \\ ENRIQUE GARCÍA VARGAS / FRANCISCA ALBA SÁNCHEZ ${ }^{5}$ / \\ MARÍA LUISA LAVADO FLORIDO ${ }^{6}$ / CANDELA SERRA GONZÁLEZ ${ }^{7}$ / \\ JOSE JUAN DÍAZ RODRÍGUEZ ${ }^{8}$ / SARA NÚÑEZ DE LA FUENTE9 / \\ LUIS GETHSEMANÍ PÉREZ AGUILAR ${ }^{10}$ / MAX LUACES ${ }^{11}$
}

\begin{abstract}
Resumen: Análisis arqueopalinológicos se llevaron a cabo en contextos del yacimiento de La Vereda (Burguillos, Sevilla), en el sur de España, ubicados cronológicamente en el siglo III d.C. en el momento de abandono hasta la actualidad del área excavada. Los datos aportados por el estudio de pólenes y microfósiles no polínicos contribuyen notablemente a detallar la función de determinadas estructuras documentadas en el sitio. En época romana se cultivaron cereales, lino y olivos, en un paisaje muy alterado por las actividades antrópicas.
\end{abstract}

\footnotetext{
1 Departamento de Geografía, Prehistoria y Arqueología, Universidad del País Vasco, c/ Francisco Tomás y Valiente s/n. 01006 Vitoria-Gasteiz. Correo-e: sebas.perezdiaz@gmail.com.

2 Departamento de Historia, Geografía y Filosofía, Universidad de Cádiz, Facultad de Filosofía y Letras. Avda. Dr. Gómez Ulla s/n. 11003 Cádiz. Correo-e: dario.bernal@uca.es.

3 Grupo de Investigación Arqueobiología, Instituto de Historia, Centro de Ciencias Humanas y Sociales, CSIC. c/ Albasanz 26-28. 28037 Madrid. Correo-e: joseantonio.lopez@cchs.csic.es.

4 Departamento de Prehistoria y Arqueología, Universidad de Sevilla, c/ Doña María de Padilla s/n. 41004 Sevilla. Correo-e: egarcia@us.es.

5 Departamento de Botánica, Universidad de Granada, Facultad de Ciencias. Avda. Fuentenueva s/n. 18071 Granada. Correo-e: falba@ugr.es

6 Arqueóloga profesional. Correo-e: marialuisa_lavado@hotmail.com.
}

Abstract: Palynological analyses were carried out on layers from the site of La Vereda in Burguillos (Sevilla), in southern Spain. The layers dated to the $3^{\text {rd }}$ century AD, around the time of the abandonment of the present excavated area. Data from pollen and non-pollen palynomorphs contributed to add details on the function of the site and their structures. During Roman times, cereals, flax and olive were cultivated, and human activities highly influenced the landscape.

7 Grupo de Investigación Arqueobiología, Instituto de Historia, Centro de Ciencias Humanas y Sociales, CSIC. c/ Albasanz 26-28. 28037 Madrid. Correo-e: sgcande@gmail.com.

8 Departamento de Historia, Geografía y Filosofía, Universidad de Cádiz, Facultad de Filosofía y Letras. Avda. Dr. Gómez Ulla s/n. 11003 Cádiz. Correo-e: josejuan.diaz@uca.es.

9 Instituto Internacional de Investigaciones Prehistóricas, Universidad de Cantabria, Avda. de los Castros s/n. 39005 Santander. Correo-e: sara.n.delafuente@gmail.com.

10 Departamento de Prehistoria y Arqueología, Universidad de Sevilla, c/ Doña María de Padilla s/n. 41004 Sevilla. Correo-e: lperez4@us.es.

11 Departamento de Historia, Geografía y Filosofía, Universidad de Cádiz, Facultad de Filosofía y Letras. Avda. Dr. Gómez Ulla s/n. 11003 Cádiz; y Universidad de Lyon. Correo-e: luacesmax@gmail.com. 
Palabras clave: Arqueopalinología, Villa Romana, Producción aceitera, Lino, Cultivo de cereales

\section{INTRODUCCIÓN}

La arqueobotánica es intrínsecamente una rama de conocimiento interdisciplinar que dispone de una alta capacidad para reconstruir tanto el paleoambiente circundante a los yacimientos arqueológicos como la economía, el estatus social y los conocimientos etnobotánicos de las culturas del pasado (Dincauze 2000). Sus evidencias empíricas se basan en la suposición de que los restos vegetales aparecen principalmente en los niveles arqueológicos debido al transporte humano, de tal manera que si algunas especies de plantas están sobrerrepresentadas en contextos específicos, su documentación detallada puede ser fiel reflejo de comportamientos y actividades humanas concretas (Mercuri 2008).

Por otro lado, la distribución espacial y la recuperación en grandes cantidades de determinados restos vegetales puede ayudar a entender cómo fueron utilizadas determinadas estructuras y contextos arqueológicos específicos (Van der Veen 2007). Mientras que los macrorrestos vegetales, particularmente semillas y frutos carbonizados, ofrecen una clara evidencia sobre la presencia de graneros y otras instalaciones de almacenamiento, así como letrinas o basureros de una manera generalizada (Bandini Mazzanti et al. 2005), existen muy pocos pero en cambio interesantísimos ejemplos que muestran cómo la señal polínica puede arrojar luz sobre el uso particular dado a ciertas estructuras o lugares (Mariotti Lippi et al. 2009; López Merino et al. 2010; Bosi et al. 2011).

El yacimiento de La Vereda (Burguillos, Sevilla) alberga un gran interés, dentro de la historiografía arqueológica andaluza, por contar con varias fases de ocupación entre el Bronce Final/Hierro I y la Antigüedad Tardía, poco conocidas a nivel paleoambiental (López García y López Sáez 1994a, 1994b). El yacimiento fue excavado con motivo de una actividad arqueológica preventiva durante los años 2013 y 2014, dirigida por M.L. Lavado Florido (2014), y actualmente se encuentra en proceso de estudio por parte de un equipo de las universidades de Cádiz y de Sevilla.

Dentro del marco cronológico en el cual se ha documentado actividad antrópica en el lugar, la fase de época romana tiene un inicio claramente atestiguado en época augustea, constatándose una presencia aparentemente continuada a lo largo de la época altoimperial y
Keywords: Archaeopalynology, Roman Villa, Oil production, Flax, Cereal cultivation

siendo muy reseñables los niveles de abandono detectados en varias zonas del área excavada, que podrían situarse en torno al segundo cuarto o mediados del siglo III d.C. (Bernal Casasola et al. 2014). En cualquier caso, el abandono definitivo del yacimiento es posterior, en momentos avanzados ya del siglo IV o inicios del siglo V d.C.

Dentro de la amplia zona excavada (más de una hectárea), se han definido distintas zonas correspondientes a un área alfarera provista de hornos y fosas de extracción de arcillas, un ambiente de molturación con al menos un molino de sangre de grandes dimensiones (figura 1A), sendos edificios separados por una calle vinculados a actividades productivas y, finalmente, varios enterramientos de una necrópolis de inhumación, de todo lo cual se ha dado un sucinto avance (Bernal Casasola et al. 2014).

Entre las estructuras más significativas de este enclave destaca un edificio cuadrangular con varias habitaciones, en alguna de las cuales se disponen piletas rectangulares que desaguaban en canalizaciones, de finalidad desconocida, ante la parquedad de evidencias aparecidas durante las excavaciones. Debido al área en la que se ubica -comarca tradicionalmente olivarera-y al hallazgo del citado molino y de los abundantes dolia y barreños de grandes dimensiones resinados (figura 2), se planteaba como muy probable la producción aceitera en este enclave romano. No obstante, no se descartaban otras actividades, como la producción textil, por el hallazgo de algunos pondera troncocónicos cerámicos durante las excavaciones.

Aunque parece evidente que las estructuras antes señaladas guardan relación con la elaboración o prensado de algún producto, cuya fracción líquida requiriese evacuación, se desconocía su utilidad concreta. Con base en ello, los objetivos que se plantearon en este trabajo fueron:

i) Reconstruir el paleopaisaje y las bases paleoeconómicas del yacimiento arqueológico de La Vereda, tanto a escala local como regional, en el marco cronológico considerado;

ii) Determinar qué productos pudieron haberse estado procesando en las instalaciones antes citadas, toda vez que no se han recuperado macrorrestos vegetales ni residuos químicos resultantes de los procesos llevados a cabo en ellas. 


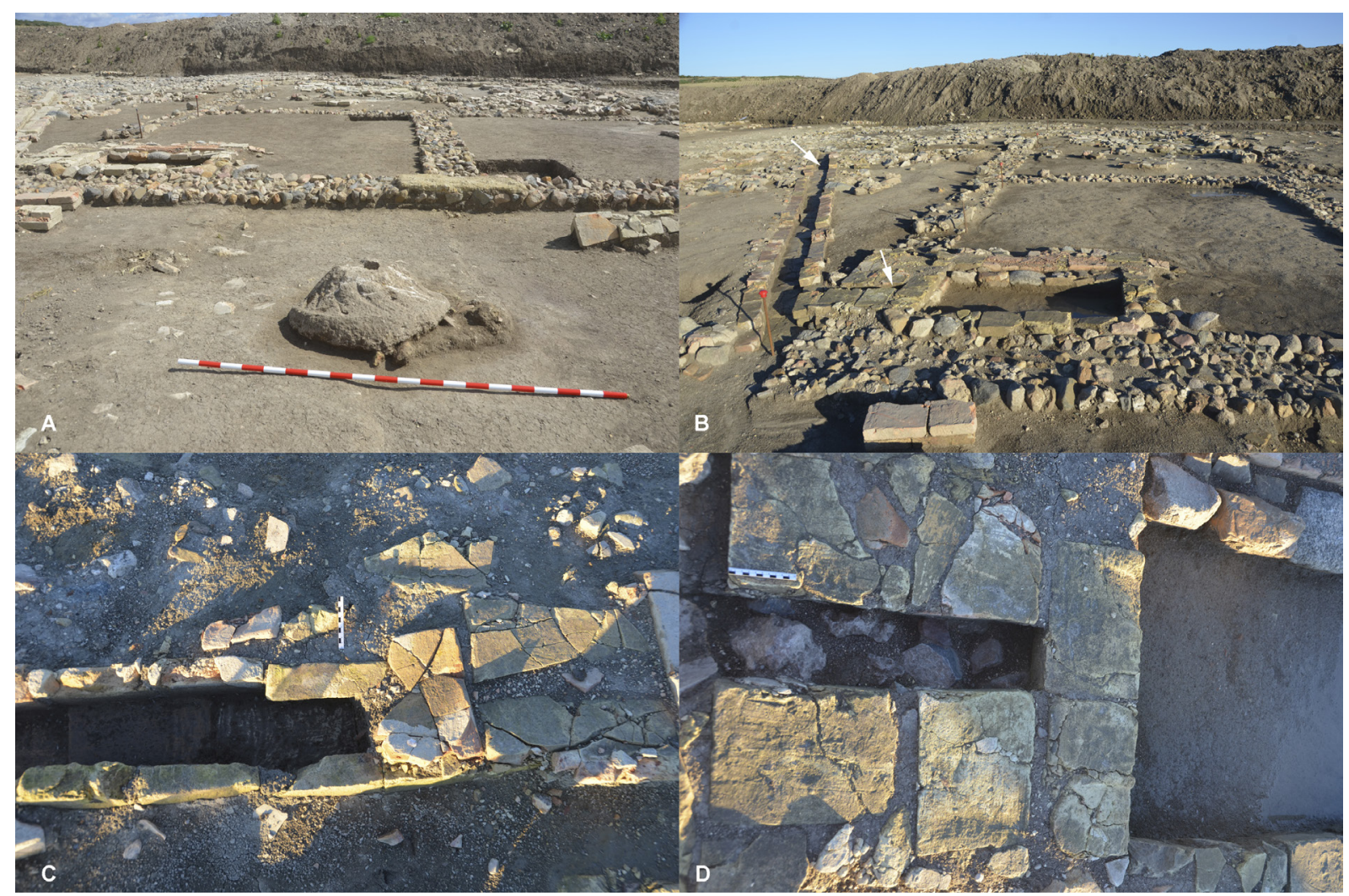

Figura 1. Vista general de las estructuras artesanales romanas de La Vereda, con la meta del molino romano in situ (A) y las canalizaciones muestreadas (B, flechas), con detalle cenital del relleno de la canalización norte (C) y del canal de desagüe de la pileta (D).

\section{METODOLOGÍA}

Las muestras palinológicas del yacimiento de La Vereda proceden de las canalizaciones aparecidas en el edificio cuadrangular y en el área adyacente (figura 1B). En estas estructuras se recogieron tres muestras para su correspondiente análisis palinológico:

- i y ii) Muestras A y B en la canalización norte, la primera de la parte inferior del relleno sedimentario de la misma que es posterior a su abandono, ya que se ha documentado con su cubierta intacta; la segunda a partir de un raspado de la parte superior del ladrillo de la base de dicha canalización (figura 1C).

- iii) Muestra $C$ en el desagüe de la pileta rectangular de la habitación meridional, a partir de los sedimentos del propio suelo de la canalización con sus adherencias (figura 1D).

El tratamiento químico de las tres muestras (10 gr de sedimento) ha sido el usual en los estudios arqueopalinológicos (Burjachs et al. 2003). Este se llevó a cabo en el Laboratorio de Arqueobiología del CCHS-CSIC, en su Unidad de Palinología. Consiste en un primer ataque al sedimento con $\mathrm{HCl}$ para la disolución de los carbonatos, seguido de $\mathrm{NaOH}$ para la eliminación de la materia orgánica, y finalmente HF para la eliminación de los silicatos. El sedimento se trató con 'licor de Thoulet' para la separación densimétrica de los microfósiles (Goeury y de Beaulieu 1979). La porción del sedimento que se obtuvo al final del proceso se conservó en gelatina de glicerina en tubos eppendorf. No se procedió a la tinción de las muestras por la posibilidad que existe de que enmascare la ornamentación de ciertos tipos polínicos. Tras el tratamiento y conservación, las muestras se montaron en portaobjetos con cubreobjetos y posterior sellado con histolaque, para proceder al recuento de los distintos tipos polínicos y no polínicos al microscopio óptico (60x, 40x).

Los morfotipos polínicos han sido establecidos según Valdés et al. (1987), Faegry e Iversen (1989), Moore et al. (1991) y Reille $(1992,1995)$. En la distinción morfológica de Oleaceae se siguió a RenaultMiskovsky et al. (1976). Los microfósiles no polínicos 

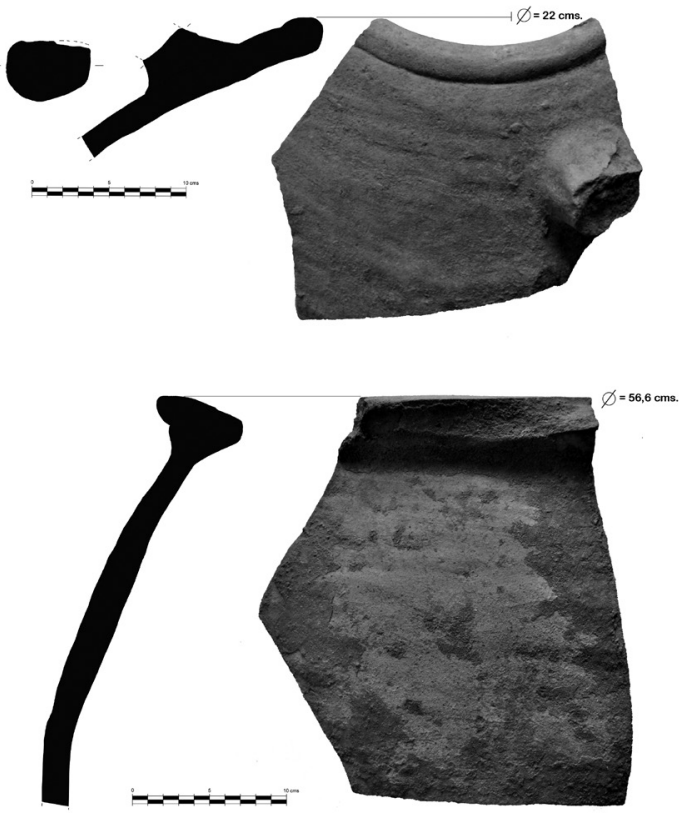

Figura 2. Dolios cerámicos de grandes dimensiones $(22$ y $56,6 \mathrm{~cm}$ de diámetro respectivamente), con resina al interior, procedentes de los niveles de abandono de las estructuras romanas de La Vereda.

se identificaron básicamente según López Sáez et al. (1998, 2000) y Van Geel (2001).

En la validación de los datos obtenidos se han aceptado las directrices estadísticas y tafonómicas expuestas en López Sáez et al. (2003, 2006, 2013). Siempre que se ha dado una muestra por válida, el número de granos de polen contados o suma base polínica (S.B.P.) ha superado los 200 procedentes de plantas terrestres, con una variedad taxonómica mínima de 20 tipos polínicos distintos. En el cálculo de los porcentajes se han excluido de la suma base polínica los taxa hidro-higrófilos y los microfósiles no polínicos, que se consideran de carácter local o extra-local, por lo que suelen estar sobrerrepresentados. Además, se han excluido de ésta Cardueae, Cichorioideae y Aster debido a su carácter antropozoógeno. El valor relativo de los palinomorfos excluidos se ha calculado respecto a la S.B.P.

\section{RESULTADOS}

En la figura 3 se representa el histograma palinológico referido al análisis polínico llevado a cabo en el yacimiento arqueológico de La Vereda, para lo cual se han empleado los programas TILIA y TGView (Grimm 1992, 2004). Los diferentes morfotipos, polínicos y no polínicos, aparecen representados de izquierda a derecha por los árboles (verde), herbáceas (amarillo), hidrohigrófitas (azul) y hongos coprófilos (marrón).

Pólenes y microfósiles no polínicos han sido documentados en las tres muestras estudiadas en un buen estado de conservación. Los 22 taxa polínicos y los 2 no polínicos están representados en todas las muestras en porcentajes relativamente parecidos, todo lo cual nos lleva a admitir la contemporaneidad de estas y el poder tratarlas conjuntamente.

\section{DISCUSIÓN E INTERPRETACIÓN}

El estudio palinológico del yacimiento de La Vereda refleja la composición del paisaje vegetal, tanto de su entorno inmediato como en un marco regional, en los primeros siglos de nuestra era. En estos momentos la cobertura arbórea es poco importante $(35,9-38,1 \%)$, menos aún si consideramos que gran parte de estos valores porcentuales hacen referencia al cultivo del olivo (Olea europea, 7,7-12,9\%).

El morfotipo polínico dominante, entre los árboles, es Quercus ilex (13,1-16,4\%). Gracias a los estudios de lluvia polínica actual (López Sáez et al. 2010), tales porcentajes $(<20 \%)$ pueden interpretarse basándose en la existencia de un paisaje local de dehesa de encinas relativamente abierto y sin cobertura arbustiva alguna. Junto a la encina aparecerían ciertos elementos termófilos de vocación rupestre (Ramón-Laca y Mabberley 2004), caso del algarrobo (Ceratonia siliqua 2,4-3,3\%), un elemento común en el Holoceno de esta zona de la provincia de Sevilla al sur de la Sierra Norte, según muestran otros análisis palinológicos realizados, caso del yacimiento de Matarrubilla en Valencina de la Concepción (Llergo y Ubera 2006). Ambos elementos florísticos, encina y algarrobo, serían característicos de la vegetación climácica del área de estudio (Rivas Martínez 1987) correspondiente a la serie termomediterránea bética seca-subhúmeda y basófila de la encina.

En un ámbito regional, la presencia de Quercus faginea con porcentajes relativamente bajos (3,3-4,6\%) atestiguaría la existencia de quejigares en depresiones umbrías y frescas, emplazadas probablemente en la cercana Sierra Norte. Por su parte, los porcentajes de pino piñonero (Pinus pinea) son igualmente bajos $(4,1-$ $5,6 \%$ ), por lo que, teniendo en cuenta el carácter anemófilo de este morfotipo y su alta producción polínica, es 


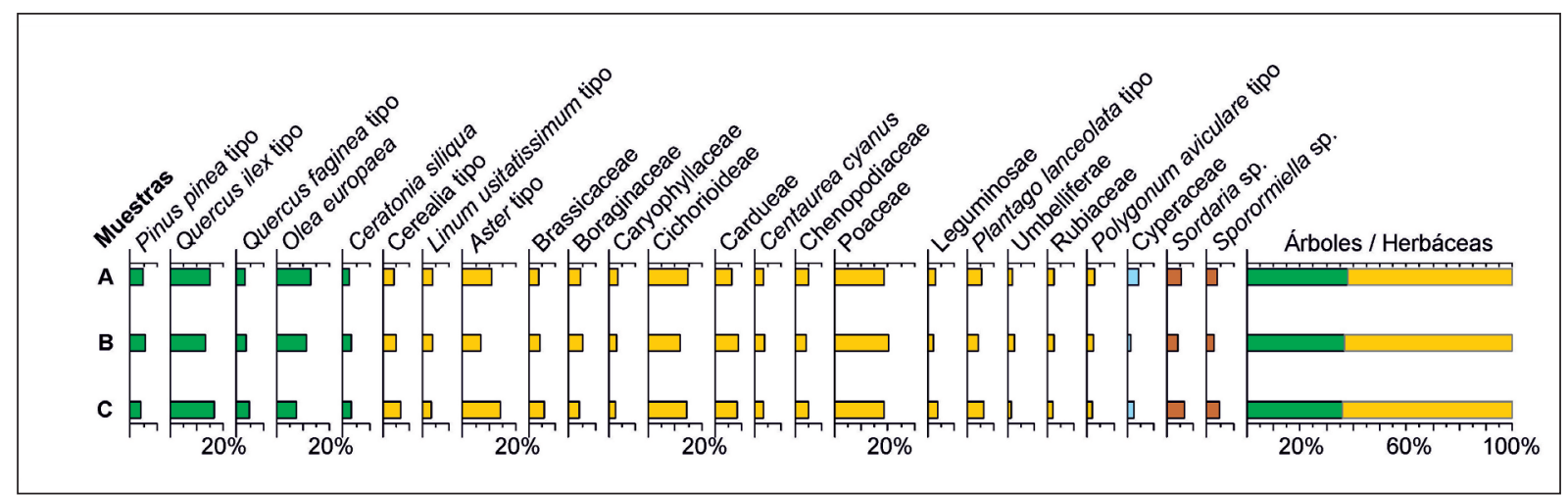

Figura 3. Histograma palinológico del yacimiento de La Vereda.

factible admitir su origen extra-regional, probablemente a partir de pinares situados en la depresión del Guadalquivir del Parque Nacional de Doñana (Martín Consuegra et al. 1998; Martínez García y Montero 2004).

Los elementos herbáceos dominan los espectros de las tres muestras analizadas $(61,9-64,1 \%)$. Dentro de estos, el mayoritario es Poaceae (18,6-20,1\%), dando cuenta de la existencia de amplias zonas aclaradas pobladas de pastizales ricos en gramíneas, a las que podrían acompañar algunos elementos de amplio espectro ecológico (Brassicaceae, Caryophyllaceae, Leguminosae, Umbelliferae) o algunos de carácter más termófilo como Rubiaceae. Conjuntamente, dentro de las herbáceas, dominan sobre todo los elementos antrópiconitrófilos (Aster: 7-14,4\%; Boraginaceae: 4,1-5,1\%; Cichorioideae: 11,7-14,8\%; Cardueae: 6,2-8,9\%). Este cortejo florístico poblaría las zonas de paso o las sometidas a un mayor impacto antrópico, donde la actividad humana directa fuera más evidente (Behre 1981).

Uno de los datos más reseñables del estudio palinológico de La Vereda es la abundancia, en sus espectros polínicos, tanto de elementos herbáceos antropozoógenos (Chenopodiaceae 4,2-5,2\%\%, Plantago lanceolata 4,2-6,2\% y Polygonum aviculare 2,1-2,9\%) como de ascosporas de hongos coprófilos que viven sobre excrementos animales (Sordaria 4,2-6,7\%, Sporormiella 2,8-4,6\%), que, en conjunto, estarían dando cuenta de una presión pastoral local, en el entorno inmediato del yacimiento, relativamente importante (López Sáez y López Merino 2007). Seguramente sería este tipo de actividad ganadera una de las razones de la fisionomía paisajística (dehesa) descrita, donde además del estrato arbóreo de encinas abundarían amplias zonas de pastizal de gramíneas con tal vocación, y cortejos florísticos antropozoógenos asociados a tal práctica. Este paisaje descrito concuerda plenamente con el documentado en otros estudios palinológicos emprendidos en la provincia de Sevilla dentro de la misma cronología (López García y López Sáez 1997; Llergo et al. 2003).

Entre las plantas cultivadas tres han sido los morfotipos identificados: Cerealia (4,3-6,7\%), Linum usitatissimum (3,1-3,8\%) y el ya mencionado Olea europaea. Centaurea cyanus (3,1-3,7\%), a tal respecto, es un buen indicador de estos cultivos como mala hierba acompañante (Vuorela 1995; Binka 2003). En cuanto a los cereales, su porcentaje es elevado, suficiente ( $>3 \%$ ) para admitir la existencia de campos dedicados a la cerealicultura en el entorno inmediatamente anexo al yacimiento (Martín Consuegra et al. 2003; López Sáez y López Merino 2005). Los valores de lino (Linum), también son altos, confirmando el cultivo de este taxón cerca del sitio así como su probable uso, en el seno de las estructuras citadas con anterioridad, bien como planta oleífera, bien como textil, toda vez que la documentación de restos vegetales de esta planta es relativamente común en contextos de época romana (Van der Veen 1996) e incluso en yacimientos andaluces prehistóricos (Peña Chocarro 2000). Finalmente, en cuanto al olivo, sus porcentajes son muy elevados (7,7-12,9\%) como para pensar en su presencia natural, por lo que todo indica su más que probable cultivo local (Laval et al. 1990; Díaz Fernández 1994), sin descartar que algunos pólenes pudieran proceder de ejemplares silvestres o acebuches presentes en las estaciones más termófilas de la comarca. En definitiva, el procesado de olivo y lino podría explicar la funcionalidad de las estructuras aquí estudiadas gracias a los datos palinológicos aportados por este estudio.

En último término, aunque anteriormente se haya admitido la naturalidad del algarrobo (Ceratonia sili$q u a)$ en el paisaje anexo al yacimiento, es cierto que sus elevados porcentajes $(>2 \%)$ también podrían suponer 
que este taxón fue de alguna manera cultivado o propagado, ya que normalmente este palinomorfo está infrarrepresentado en los espectros polínicos (Jahns 2003), y tal y como señala Zohary (2002) fue una especie ampliamente cultivada en la cuenca mediterránea a partir de la época romana.

\section{Agradecimientos}

El trabajo palinológico ha sido financiado por el Proyecto de investigación de Excelencia P11RNM-7033 de la Junta de Andalucía; y la parte arqueológica por el proyecto HAR2013-43599P y HAR2016-78691P del MINECO.

\section{BIBLIOGRAFÍA}

Bandini Mazzanti, M.; Bosi, G.; Mercuri, A.M.; Accorsi, C.A. y Guarnieri, C. (2005): "Plant use in a city in Northern Italy during the Late Medieval and Reinaissance periods: results of the Archaeobotanical Investigation of 'The Mirror Pit' (14th-15th century A.D.) in Ferrara". Vegetation History and Archaeobotany 14: 442-452.

Behre, K.E. (1981): "The interpretation of anthropogenic indicators in pollen diagrams". Pollen et Spores 23: $225-245$.

Bernal Casasola, D.; García Vargas, E.; Lavado Florido, M.L.; Díaz Rodríguez, J.J.; Luaces, M. y Pérez Aguilar, L.G. (2014): “M. Petrucidius y los hornos romanos y canteras de extracción de arcilla de La Vereda (Burguillos, Sevilla)". Boletín Ex Officina Hispana 5: 26-31.

Binka, K. (2003): "Palynological evidence for plantanimal interaction in the late Holocene". Vegetation History and Archaeobotany 12: 37-47.

Bosi, G.; Bandini Mazzanti, M.; Florenzano, A.; Massamba, I.; Pederzoli, A.; Rinaldi, R.; Torri, P. y Mercuri, A.M. (2011): "Seeds/fruits, pollen and parasite remains as evidence of site function: piazza GaribaldiParma (N Italy) in Roman and Mediaeval times". Journal of Archaeological Science 38: 1621-1633.

Burjachs, F.; López Sáez, J.A. e Iriarte, M.J. (2003): "Metodología Arqueopalinológica", en R. Buxó y R. Piqué (eds.), La recogida de muestras en Arqueobotánica: objetivos y propuestas metodológicas. La gestión de los recursos vegetales y la transformación del paleopaisaje en el Mediterráneo occidental: 1118. Barcelona, Museu d'Arqueologia de Catalunya.
Díaz Fernández, P. (1994): "Relations between modern pollen rain and mediterranean vegetation in Sierra Madrona (Spain)". Review of Palaeobotany and Palynology 82: 113-125.

Dincauze, D.F. (2000): Environmental Archaeology: Principles and Practice. Cambridge, Cambridge University Press.

Faegri, K. e Iversen, J. (1989): Textbook of Pollen Analysis. Chichester, John Wiley \& Sons.

Goeury, C. y de Beaulieu, J.L. (1979): “À propos de la concentration du pollen à l'aide de la liqueur de Thoulet dans le sédiments minéraux". Pollen and Spores 21: 239-251.

Grimm, E.C. (1992): Tilia, version 2. Springfield, Illinois State Museum.

Grimm, E.C. (2004): TGView. Springfield, Illinois State Museum.

Jahns, S. (2003): “A late Holocene pollen diagram from the Megaris, Greece, giving possible evidence for cultivation of Ceratonia siliqua L. during the last 2000 years". Vegetation History and Archaeobotany 12: 127-130.

Lavado Florido, M.L. (2014): Memoria Preliminar del Control Arqueológico de los movimientos de tierra en el Proyecto de Conducción de conexión del canal del VIAR en el sistema de abastecimiento a Sevilla. Original inédito depositado en la Delegación Territorial de la Consejería de Cultura de la Junta de Andalucía en Sevilla.

Laval, H.; Leveau, P.; Marinval, P. y Medus, J. (1990): 'L'olivier et sa culture en Provence. Données historiques, palynologiques et carpologiques". Ecologia Mediterranea 16: 427-435.

Llergo, Y. y Ubera, J.L. (2006): "Estudio palinológico del yacimiento arqueológico de Matarrubilla, Valencina de la Concepción (Sevilla)". Polen 16: 29.

Llergo, Y.; Ubera, J.L. y Román, J.M. (2003): “Estudio palinológico del complejo hipogeo de la calle de San Felipe 1A: galería D, Carmona (Sevilla)". Polen 13: 109-128.

López García, P. y López Sáez, J.A. (1994a): “El paisaje andaluz en la Prehistoria: datos paleopalinológicos". Revista Española de Micropaleontología 26: 49-59.

López García, P. y López Sáez, J.A. (1994b): “Comparison of peats and archaeological samples in the Andalusian region, Spain", en O.K. Davis (ed.), Aspects of archaeological palynology: methodology and applications: 127-139. Houston, AASP.

López García, P. y López Sáez, J.A. (1997): “Contribución al conocimiento de la historia de la vegetación 
de la provincia de Sevilla: análisis polínico del yacimiento arqueológico de Los Molares". Mediterranea. Serie de Estudios Biológicos 16: 19-22.

López Merino, L.; Peña Chocarro, L.; Ruiz Alonso, M.; López Sáez, J.A. y Sánchez Palencia, J. (2010): "Beyond nature: The management of a productive cultural landscape in Las Médulas area (El Bierzo, León, Spain) during pre-Roman and Roman times". Plant Biosystems 144: 909-923.

López Sáez, J.A.; Alba Sánchez, F.; López Merino, L. y Pérez Díaz, S. (2010): "Modern pollen analysis: a reliable tool for discriminating Quercus rotundifolia communities in Central Spain". Phytocoenologia 40: 57-72.

López Sáez, J.A.; Burjachs, F. y López Merino, L. (2006): "Algunas precisiones sobre el muestreo e interpretación de los datos en Arqueopalinología". Polen 15: 17-29.

López Sáez, J.A.; Iriarte Chiapusso, M.J. y Burjachs, F. (2013): “Arqueopalinología”, en M. García-Diez y L. Zapata (eds.), Métodos y técnicas de análisis y estudio en Arqueología Prehistórica. De lo técnico a la reconstrucción de los grupos humanos: 273290. Vitoria, Universidad del País Vasco.

López Sáez, J.A.; López García, P. y Burjachs, F. (2003): “Arqueopalinología: Síntesis crítica". Polen 12: 5-35.

López Sáez. J.A. y López Merino, L. (2005): “Precisiones metodológicas acerca de los indicios paleopalinológicos de agricultura en la Prehistoria de la Península Ibérica". Portugalia 26: 53-64.

López Sáez, J.A. y López Merino, L. (2007): “Coprophilous fungi as a source of information of anthropic activities during the Prehistory in the Amblés Valley (Ávila, Spain): the archaeopalynological record". Revista Española de Micropaleontología 39: 103-116.

López Sáez, J.A.; Van Geel, B.; Farbos-Texier, S. y Diot, M.F. (1998): "Remarques paléoécologiques à propos de quelques palynomorphes non-polliniques provenant de sédiments quaternaires en France". Revue de Paléobiologie 17: 445-459.

López Sáez, J.A.; Van Geel, B. y Martín Sánchez, M. (2000): “Aplicación de los microfósiles no polínicos en Palinología Arqueológica", en V. Oliveira Jorge (ed.), Contributos das Ciências e das Technologias para a Arqueologia da Península Ibérica, Actas $3^{\circ}$ Congresso de Arqueología Peninsular, vol. IX: 11-20. Oporto, Adecap.

Mariotti Lippi, M.; Bellini, C.; Mori Secci, M. y Gonnelli, T. (2009): “Comparing seeds/fruits and pollen from a Middle Bronze Age pit in Florence (Italy)". Journal of Archaeological Science 36: 1135-1141.

Martín Consuegra, E.; Chisvert, N.; Cáceres, L. y Ubera, J.L. (1998): “Archaeological, palynological and geological contributions to landscape reconstruction in the alluvial plain of the Guadalquivir river at San Bernardo, Sevilla (Spain)". Journal of Archaeological Science 25: 521-532.

Martín Consuegra, E.; Ubera, J.L. y Romo, A. (2003): "Estudio palinológico del yacimiento arqueológico de la Plaza Virgen de Los Reyes, Sevilla". Polen 13: 143-154.

Martínez García, F. y Montero, G. (2004): “The Pinus pinea L. woodlands along the coast of South-western Spain: data for a new geobotanical interpretation". Plant Ecology 175: 1-18.

Mercuri, A.M. (2008): "Plant exploitation and ethnopalynological evidence from the Wadi Teshuinat area (Tadrart Acacus, Libyan Sahara)". Journal of Archaeological Science 35: 1619-1642.

Moore, P.D.; Webb, J.A. y Collinson, M.E. (1991): Pollen analysis. Londres, Blackwell Scientific Publications.

Peña Chocarro, L. (2000): “Agricultura y alimentación vegetal en el poblado de la Edad del Bronce de Peñalosa (Baños de la Encina, Jaén)". Complutum 11: 209-219.

Ramón-Laca, L. y Mabberley, D.J. (2004): “The ecological status of the carob-tree (Ceratonia siliqua, Leguminosae) in the Mediterranean". Botanical Journal of the Linnean Society 144: 431-436.

Reille, M. (1992): Pollen et Spores d'Europe et d'Afrique du Nord. Marsella, Laboratoire de Botanique Historique et Palynologie.

Reille, M. (1995): Pollen et Spores d'Europe et d'Afrique du Nord. Supplement 1. Marsella, Laboratoire de Botanique Historique et Palynologie.

Renault-Miskovsky, J.; Girard, M. y Trouin, M. (1976): “Observations de quelques pollens d'Oléacées au microscope électronique à balayage". Bulletin de l'Association française pour l'Étude du Quaternarie 2: 71-86.

Rivas Martínez, S., (1987): Memoria del MAPA de Series de Vegetación de España, 1: 400.000. Madrid, M.A.P.A.-I.C.O.N.A.

Valdés, B.; Díez, M.J. y Fernández, I. (1987): Atlas polínico de Andalucía Occidental. Sevilla, Universidad de Sevilla - Diputación de Cádiz.

Van der Veen, M. (1996): "The plant remains from Mons Claudianus, a Roman quarry settlement in the Eastern Desert of Egypt - an interim report". Vegetation History and Archaeobotany 5: 137-141. 
Van der Veen, M. (2007): "Formation processes of desiccated and carbonized plant remains and the identification of routine practice". Journal of Archaeological Science 34: 968-990.

Van Geel, B. (2001): "Non-pollen palynomorphs", en J.P. Smol; H.J.B. Birks y W.M. Last (eds.), Tracking environmental change using lake sediments. Volume
3: Terrestrial, Algal, and Siliceous Indicators: 99119. Dordrecht, Kluwer Academic Publishers.

Vuorela, I. (1995): "Centaurea cyanus in urban cultural layers in Southern Finland". PACT 42: 14-18.

Zohary, D. (2002): "Domestication of the Carob (Ceratonia siliqua L.)". Israel Journal of Plant Sciences 50: 141-145. 